\title{
Real-time Tumor Tracking with Respiratory Motion Based on Short-term Prediction
}

\author{
Tian Qiao", Yixu Song ${ }^{1, *}$ and Chao Ren ${ }^{2}$ \\ ${ }^{1}$ State Key Laboratory on Intelligent Technology and Systems \\ Tsinghua National Laboratory for Information Science and Technology \\ Department of Computer Science and Technology, Tsinghua University, Beijing 100084, China \\ ${ }^{2}$ Department of Interventional Ultrasound, Chinese PLA General Hospital, 28 Fuxing Road, Beijing, 100853, China \\ ${ }^{*}$ Corresponding author
}

\begin{abstract}
The purpose of this study is to design a 3D navigation strategy with 2D ultrasonic images, with the assumption that the internal target trajectory could be evaluated with extern surrogate signals. This paper first proposes a simple 3D navigation strategy and then designs a fast tumor tracking system that exploits learning based methods, which learns the mapping relation between the external surrogate signals and the internal tumor trajectory. This paper uses our own retrospective clinical data to test the developed system. The experimental results show that this system has the potential to implementing high accuracy tumor tracking and navigation.
\end{abstract}

Keywords-learning-based; tumor tracking; surrogate signals; respiratory motion.

\section{INTRODUCTION}

\section{A. Motivation}

In interventional ablation surgery, radiofrequency or microwave is commonly used to inactivate malignant cells to treat cancer. Ablation therapy is actually a palliative, curative and adjuvant treatment strategy. Its operating principle is to take the highest toll on the tumorous target while preserving surrounding healthy tissues. However, tumor position in the epigastric areas are often affected by physiological activities such as respiration, gastrointestinal peristalsis, and heartbeat. Indeed, average craniocaudal displacement of liver could reach $10 \mathrm{~mm}$ to $25 \mathrm{~mm}$ for shallow breadth, and $37 \mathrm{~mm}$ to $55 \mathrm{~mm}$ for deep breadth $[1,2,3]$, far higher than the tolerable precision (millimeter scale) of clinical surgeries. FIGURE I. shows a clear respiratory motion effect on liver position. In these cases, without any navigation assistance, uncertainties of target positioning force surgeon to expand the planning treatment volume around the real target volume, which increases the volume of healthy tissues being inactivated. Therefore, real-time tumor tracking system plays a significant role in ablation surgery.

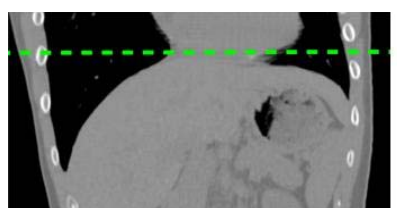

(a) deep inhale

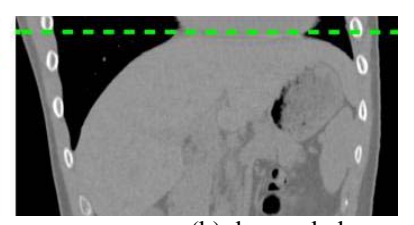

(b) deep exhale
FIGURE I. THE UPPER-ABDOMINAL ORGAN POSITION AT THE END OF INSPIRATION AND EXPIRATION.

\section{B. Related Works}

The internal motion can be directly visualized in radiation therapy and ultrasound (US) guided surgery. For radiation therapy, one or more metallic markers are optionally implanted inside or near the target, then their positions can be tracked in real-time with fluoroscopic imaging device [4]. Real Time Radiation Therapy [5] continuously detects and recognizes the internal target position. However, the obvious weakness of this method consists in the extra non-therapeutic dose patients received during the fluoroscopic acquisition. For ultrasoundguided surgery, the poor quality but real-time ultrasound images are useful for doctors to track the tumor under respiration. However, it's challenging to navigate the surgery in $3 \mathrm{D}$ space with the $2 \mathrm{D}$ images.

Also, external motion can be exploited as a surrogate of the internal motion, during either the 4DCT reconstruction [6] or the treatment delivered by means of tracking techniques. The displacement of the epigastric surface is commonly detected with the depth-based camera [7], respiration belt [8], and mostly infra-red devices [3,9]. For example, the RPM system can monitor the respiration by locating a cube placed on the patient's abdomen [10]. However, for the purpose of capturing the movements as comprehensive as possible, multiple markers should be placed on the thorax and/or abdomen of the subject.

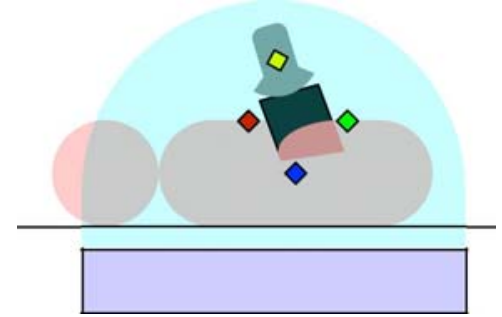

FIGURE II. THE TRAINING SCENARIO: THE YELLOW, RED, GREEN AND BLUE DOTS REPRESENT MAGNETIC SENSORS, WHICH SHOULD BE DROWN IN THE FIELD DRAWN AS THE CIEL REGION. THE GRAY RECTANGLE INDICATES THE ULTRASOUND (US) PLANE, WHILE LIGHT GRAY OBJECT INDICATES US PROBE.

It's necessary to correlate the external motion and the tumor position for real-time tumor localization based on external signals. Various prediction methods have been researched for the localization problem with intra-fractional variations. Linear predictive models $[11,12]$ are used for estimating the future 
tumor position based on the linear accumulation of extern signals. In view of the nonlinear respiratory motion, Kalman Filter was adapted for introducing weak nonlinearity into predictive model $[11,13,14,15]$. But, the heavy computation load and weak linearity limit their further application. Next, artificial neural network and its extended approaches have advanced the prediction performance greatly despite its timeconsuming computation $[16,12,17,18,19]$.

\section{Challenges}

The ideal localization system for interventional ablation treatment should be equipped with following qualities: (a) The system should decrease the amount of non-therapeutic radiation dose on patient. (b) The developed method should provide similar accuracy in locating tumor with respect to the fluoroscopic methods. (c) This system should be able to acquire and process data with less computing time for real- time processing. (d) The system should be affordable for wide deployment and open to third-party integration.

\section{Contributions}

In this paper, we propose a real-time abdominal tumor localization system for intra-fraction variations based on the correlation between internal target and external marker status. The gradient boosting method is adapted for estimating the mutual information between external markers and internal tumor position. Moreover, we propose a new method for selection of the layout of the external surrogate signals based on the feature selection techniques. The electromagnetic positioning system is adapted instead of optical localization device, which leaves surgeon more freedom for operation without worrying about veiling light. The overall framework works as follows: First, acquire the tumor position by medical image analysis automatically. Commonly, the orthogonal X-ray images or ultrasound images are frequently used for specific scenarios. In this paper, we extract target position inside liver by analyzing ultrasound images. Then, train the correlation model with external signals as input features, and tumor position as labels. During treatment, this paper use GBM to output the tumor position, according to the simultaneous status of external markers. The experiment show that our system can reach a relatively high accuracy with less computing time.

\section{ForeCASTING Methodology}

In this section, we first introduce the data collecting and processing, including label extraction and data quality control. Then, we provide a nonparametric formalization of our model. Next, we briefly review gradient boosting method and describe how stability selection can be incorporated to control for false positives, followed by the discussion of the properties of GBM

\section{A. Data Preprocessing}

In our system, we localize surface markers and surgical tools with electromagnetic localization system and its coordinate is treated as the world coordinate. The reasons why we use the magnetic based localization system are listed as follows: First, it is open to integrate third-party instruments like X-ray devices, ultrasound devices, CT devices, et cetera. Second, the system is robust to optical shield. Third, is affordable for wide deployment compared with fluoroscopic localization system.

1) Label Extraction: Before surgery, the ultrasound images of ROI should be collected. Under the supervision of doctors, the target position can be extracted by the template matching, and then be recorded for later training. At the same time, the position and orientation of external points were also collected through electromagnetic localization system.

2) Device Calibration: Usually, the obtained target position in image coordinate is not the exact answer needed. The conversion from image coordinate to world coordinate should be completed in practice. For example, we need to calibrate every pixel in image coordinate to electromagnetic coordinate depicted in FIGURE III. Both circles in rectangle A and B represent sensors stick to the ultrasound probe. The orientation of $A$ is placed parallel to $X_{E M}$ axis when the $X_{U S} O_{U S} Y_{U S}$ is parallel to the $\mathrm{X}_{\mathrm{EM}} \mathrm{Y}_{\mathrm{EM}} Z_{\mathrm{EM}}$, the $\mathrm{O}_{\mathrm{EM}} \mathrm{X}_{\mathrm{EM}}$ is parallel to $\mathrm{O}_{\mathrm{US}} \mathrm{X}_{\mathrm{US}}$, and the AOUs is already known to us. Therefore, the target position $P$ in world coordinate could be represented as

$$
P=\boldsymbol{p}+A O_{U S}+O_{U S} P=\boldsymbol{p}+A O_{U S}+(h, w, 0),
$$

where $\boldsymbol{p}$ is the sensor position in world coordinate, and $(h, w)$ is the target position in image coordinate.

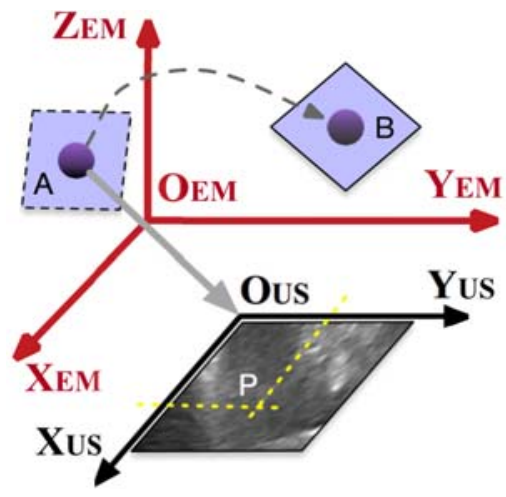

FIGURE IV. DIAGRAM OF CALIBRATING ULTRASONIC IMAGE. THE $\mathrm{X}_{\text {uS }} \mathrm{O}_{\text {us }} \mathrm{Y}_{\text {us }}$ DENOTES THE 2D ULTRASOUND IMAGE COORDINATE, WHILE THE $X_{\text {EM }} Y_{\text {EM }} Z_{\text {EM }}$ DENOTES THE 3D WORLD COORDINATE. THE RECTANGLE A IS THE INITIAL POSE OF US PROBE, AND RECTANGLE B IS THE CURRENT POSE OF US PROBE.

From electromagnetic field system, we can easily get the sensor position and quaternion $[\boldsymbol{p}, \boldsymbol{q}]$, where $\mathrm{p}$ is the position in world coordinate and $\mathrm{q}$ is the quaternion. Assume the data of $A$ and $B$ are $\left[\boldsymbol{p}_{\boldsymbol{A}}, \boldsymbol{q}_{\boldsymbol{A}}\right]$ and $\left[\boldsymbol{p}_{\boldsymbol{B}}, \boldsymbol{q}_{\boldsymbol{B}}\right]$, then we can address that When the sensor $A$ is moved, and rotated to the position of $B$, then its corresponding $P_{\text {new }}$ could be

$$
P_{\text {new }}=M\left(\boldsymbol{q}_{\boldsymbol{B}}-\boldsymbol{q}_{\boldsymbol{A}}\right) \cdot P+\left(\boldsymbol{p}_{\boldsymbol{B}}-\boldsymbol{p}_{\boldsymbol{A}}\right)
$$

where $M(\cdot)$ denotes the transform from quaternion to rotate matrix. Let $\boldsymbol{q}=a+b \boldsymbol{i}+c \boldsymbol{j}+d \boldsymbol{k}$, where $\boldsymbol{i}^{2}=\boldsymbol{j}^{2}=\boldsymbol{k}^{2}=$ $-1, \boldsymbol{i} \cdot \boldsymbol{j}=\boldsymbol{k}, \boldsymbol{i} \cdot \boldsymbol{k}=\boldsymbol{j}, \boldsymbol{j} \cdot \boldsymbol{k}=\boldsymbol{i}$, then the rotation matrix can be formalized as 


$$
M(\boldsymbol{q})=\left[\begin{array}{ccc}
1-2\left(c^{2}+d^{2}\right) & 2(b c-d a) & 2(b d+c a) \\
2(b c+d a) & 12\left(b^{2}+d^{2}\right) & 2 c d b a \\
2 b d c a & 2(c d+b a) & 12\left(a^{2}+c^{2}\right)
\end{array}\right]
$$

Accordingly, every pixel in ultrasonic images could be mapped into $3 \mathrm{D}$ coordinate through translation and rotation information between $A$ and $B$.

\section{B. Formalization}

We use nonparametric additive models for forecasting the tumor position. These models have non-linear relationships. In particular, the proposed models allow nonlinear and parametric terms using the framework of additive models. The predicted position against certain feature values is modeled with

$$
\boldsymbol{y}=F(\boldsymbol{x})+\boldsymbol{e}
$$

where $\boldsymbol{y}=[x, y, z]$ denotes the position of the target tumor against the input features $\boldsymbol{x}$. The $\boldsymbol{x}$ is a dimensional vector containing all positions and orientations of the selected thoracic-abdominal surface points which are considered in the model. The $\boldsymbol{e}=\left[e_{x}, e_{y}, e_{z}\right]$ denotes the model error for the input $\boldsymbol{x}$. Our goal is to estimate the function $F: \mathbb{R}^{d} \rightarrow \mathbb{R}^{3}$ for a given quadratic loss function. Therefore, the estimation of the function $F=\left\{F_{t}\right\}, t \in\{x, y, z\}$ based on a sample dataset $\left\{\boldsymbol{x}_{\boldsymbol{i}}, \boldsymbol{y}_{\boldsymbol{i}}\right\}_{i=1}^{N}$ reduces to minimizing

$$
\hat{F}=\underset{F}{\operatorname{argmin}} \frac{1}{N} \sum_{i=1}^{N}\left(\boldsymbol{y}_{\boldsymbol{i}}-F\left(\boldsymbol{x}_{\boldsymbol{i}}\right)\right)^{2}
$$

\section{Gradient Boosting Machine}

Boosting is an efficient supervised learning algorithm created with the idea of developing a strong learner by combing weak learners. Boosting methods have drawn lots of attention since it was proposed in 1990 [20, 21, 22], and keeps active in wide range of research fields and applications considering its excellent performance [23]. There is a popular believe that the ability of anti-overfitting is the key to interpreting its great performance and broad adaptability [24]. Gradient boosting estimates $F$ iteratively. We let

$$
\hat{F}^{m}(\boldsymbol{x})=\hat{F}^{m-1}(\boldsymbol{x})+\eta \hat{h}^{m}\left(\boldsymbol{x}, \hat{\theta}^{(m)}\right)
$$

where $\hat{h}^{m}(\boldsymbol{x})$ is the weak learner estimate at the $m$ th stage with its parameters $\theta^{(m)}$, and $\eta \in[0,1]$ is the decay coefficient. Since the estimation $\hat{F}^{m-1}(\boldsymbol{x})$ has been provided, we can first obtain the $\hat{h}^{m}(\boldsymbol{x})$ by computing the steepest gradient $\mu^{m}=y-$ $\hat{F}^{m-1}(\boldsymbol{x})$. Then, a regressor is feed with $\left\{\mu^{m}, \boldsymbol{x}\right\}_{t=1}^{T}$ with weak learner, shown as follows:

$$
\hat{\theta}^{(m)}=\underset{\theta}{\operatorname{argmin}} \frac{1}{N} \sum_{t=1}^{T}\left|\mu^{m}-\hat{h}^{m}(\boldsymbol{x}, \hat{\theta})\right|^{2}
$$

where $\hat{h}^{m}(\boldsymbol{x})$ is optimized to best predict the residuals with the previous model $\hat{F}^{m-1}(\boldsymbol{x})$. Finally, we can obtain the solution

$$
F(\boldsymbol{x})=\hat{F}^{(M)}(\boldsymbol{x})=\hat{h}_{0}(\boldsymbol{x})+\sum_{m=1}^{M} \eta \hat{h}^{m}(\boldsymbol{x})
$$

and the estimation could be improved continuously by adding the complement part $\eta \hat{h}^{m}(\boldsymbol{x})$ at every new stage. We can also control the overfitting degree by limiting the value of $M$. The two parameters $\eta$ and $\mathrm{M}$ are not independent to each other. The decrease of $\eta$ could increase the value of $M$. According to Friedman's work [22], smaller $\eta$ is better for controlling overfitting during boosting training. Here we set $v=0.1$. Therefore, the $\mathrm{M}$ is only parameter that needs to be optimized.

\section{EXPERIMENTS}

\section{A. Devices}

As shown in FIGURE IV, there is an electromagnetic field generator placed inside the self-designed operation table. The operation table is not magnetic sensitive so that the electromagnetic field could pass through nondisruptively. The yellow rectangle shows the system control unit (SCU) that supports 8 ports, which means we can collect position and orientation of at most 8 points simultaneously. The Aurora Electromagnetic Tracking Systems is open to integrate thirdparty developed tools. Therefore, it's easier to work with the existing devices and surgical tools. Here, we integrate the thirdparty ultrasound device into this system as shown in the green rectangle just by attaching a magnetic sensor and calibrating with necessary parameters as depicted in FIGURE III(a). As a consequence, the ultrasound images could be directly mapped into 3D magnetic coordinate.

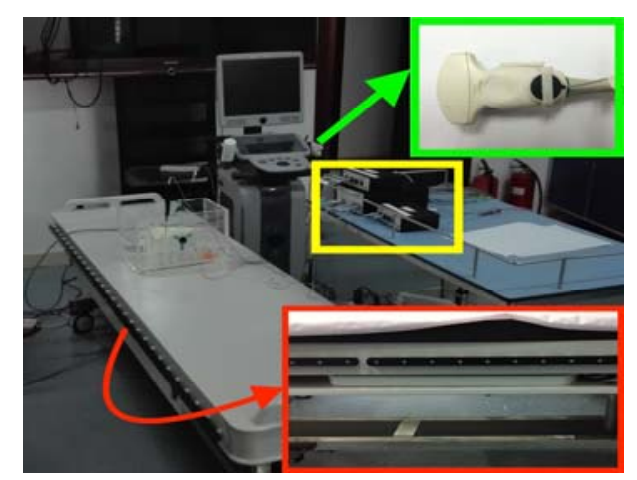

FIGURE VI. EXPERIMENTAL DEVICES: THE GREEN RECTANGLE

SHOWS THE THIRD-PARTY US PROBE ATTACHED WITH A

MAGNETIC SENSOR. THE RED RECTANGLE SHOWS THE

ELECTROMAGNETIC FIELD GENERATOR EMBEDDED IN THE

CUSTOMIZED SURGERY TABLE. ALL MAGNETIC SENSORS SHOULD BE CONNECTED TO SYSTEM CONTROL UNIT (8 PORTS) IN YELLOW RECTANGLE.

\section{B. Data Collection and Calibration}

The real-time tumor positions are collected semiautomatically. First, the surgeon marked the center of tumor region as seed point from the real-time medical image. Second, the tumor tracking algorithm tracking the seed point and record 
its absolute position. Third, we calibrate pixels in 2D image coordinate system to a $3 \mathrm{D}$ position in the electromagnetic coordinate. As shown in Fig. V, the green cross marks a kidney point as the target instead of the tumor. The selected point always has a stable and clear edge in ultrasonic images. Here we use this point as a substitution of a tumor to validate the feasibility of our method.

In FIGURE V, the doctor select a point on diaphragm as a seed point marked yellow. Then the nearby region is chosen as the template. The algorithm will find the best matching point nearby when every new frame comes. We highlight the diaphragm (the white part) so that we can see the spatial relationship between seed point and the diaphragm. FIGURE VII shows the absolute position of the selected fiducial point (instead of tumor) inner the subjective liver. This point has repetitive or quasi-repetitive motions on $\mathrm{X}$-axis, $\mathrm{Y}$-axis, and $\mathrm{Z}$ axis, meanwhile, these motions have different periodic phases.

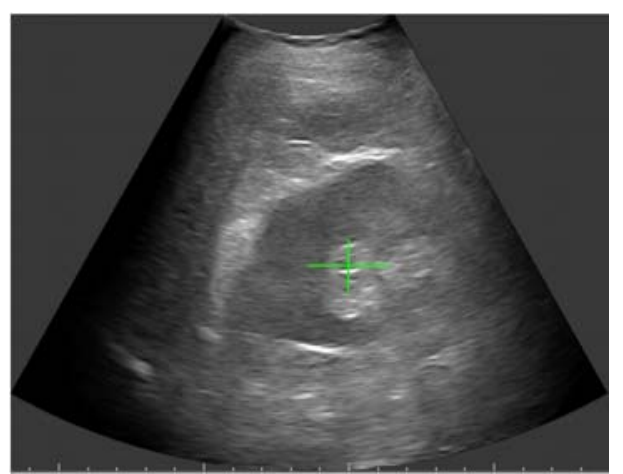

FIGURE V. TRACKING TARGET POINT IN US IMAGES SEQUENCE FOR ABOUT 1.1S BY TEMPLATE MATCHING. THE YELLOW POINT IS THE TARGET SELECTED BY THE DOCTOR. THE BLACK RECTANGLE INSIDE EVERY PICTURE INDICATES THE REGION OF INTEREST.

Then, the doctor uses the ultrasonic device to track the target point. Meanwhile, the tracking algorithm will record the position of the target according to local similarity. The algorithm will find the best matching point in the nearby region when every new frame comes. The selected point inner kidney moves periodically due to the regular respiratory motion. The z-axial value always equals to zero because the pixel is in the 2D coordinate. Besides the position of the target, the probe's simultaneous position and orientation will also be recorded with the aid of electromagnetic sensor stuck to this ultrasonic probe. Then we can easily calibrate every pixel of ultrasonic images into the 3D electromagnetic coordinate according to FIGURE III. The corresponding target trajectory in world coordinate is shown in FIGURE VI. We can find the target point inner kidney moves mainly in superior-inferior direction, which is represented with $\mathrm{Y}$-axis. This paper uses this 3D trajectory as the ground truth to train and validate prediction model.

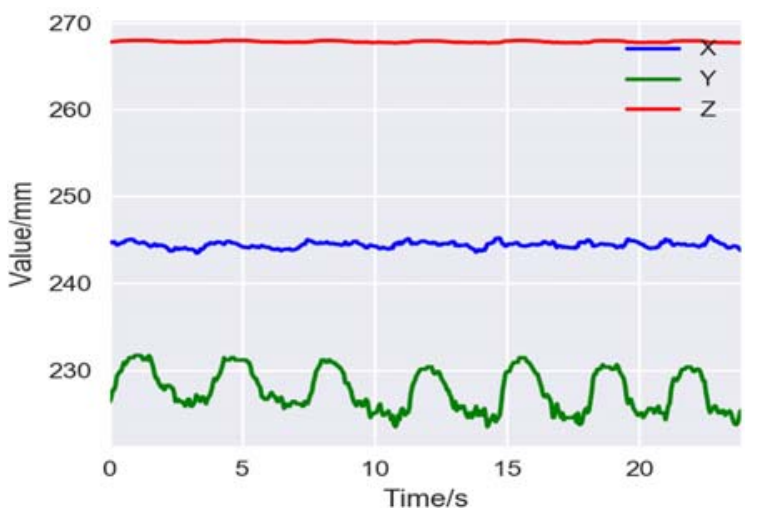

FIGURE VIII. THE REAL-TIME POSITION OF THE FIDUCIAL POINT INNER KIDNEY. THE BLUE, GREEN AND BLUE LINES INDICATES THE X-AXIAL, Y-AXIAL AND Z-AXIAL POSITION OF THE 3D EM COORDINATE RESPECTIVELY.

\section{Training and Validation Model}

In this experiment, there are 7 external sensors are clinging to patient's thoracic-abdominal surface, where 7 parameters are provided for each single sensor. Therefore, we have 49 features in total to analyze the respiration motion. Considering the limited data that we collect, we use cross-validation as the benchmark to optimizing our model. Then, we use test dataset to evaluate the performance of prediction model. By this way, we not only find out the prediction precision, but also find out the precision changing trend with the time. As we can see in FIGURE IX, the prediction of our model according to the external surrogates matches the labels perfectly. The maximum estimation error is less than $1.9 \mathrm{~mm}$ since model trained within $15 \mathrm{~s}$. The longer interval from the model trained to the current frame is, the lower prediction precision we can get. So, we must update our model with new data frame for assuring the prediction performance.

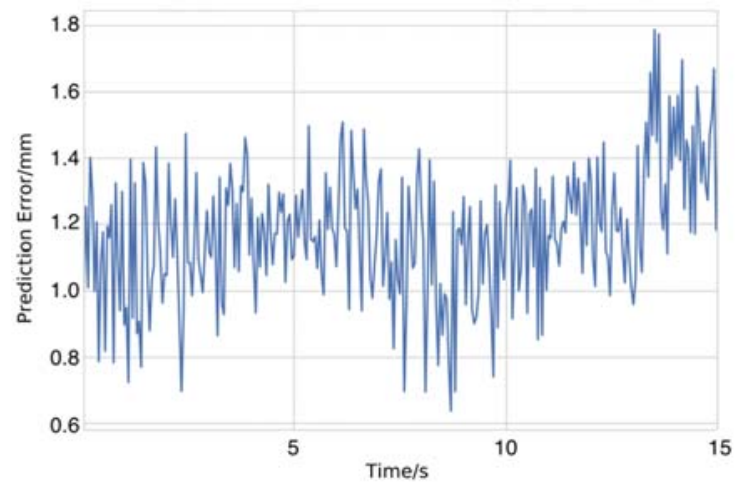

FIGURE VII. THE PREDICTIONS ERROR OF THE TARGET POSITION.

\section{CONCLUSION}

In this paper, this study shows that gradient boosting could be a helpful tool to localize the inner target with external signals as input, meanwhile validating the feasibility of modeling the relation of internal and external signals. Moreover, the integration of GBM into an electromagnetic (EM) tracking system can bring about several advantages: 
- $\quad$ GBM is an efficient supervised learning method. Its powerful anti-overfitting ability and rapid convergence could guarantee the great performance and robustness of this system.

- $\quad$ EM tracking system can track multiple external points simultaneously, but not with the optical-based system. More external points could do help to get the big picture of external respiratory motion.

- $\quad$ EM tracking system has more powerful anti-occluding ability than optical-based localization system. The surgeon no longer needs to pay attention to expose the positioning tool to the optical camera during operation.

\section{FUTURE WORK}

There are still some limitations in this work that should be reinforced in the future: (a) The number of tested subjects is not enough and more experiment should be added later; (b) The valid time for model tells surgeon the period of validity when the model is reliable. To ensure the surgery goes safely and smoothly, the valid time should be tested in the future; (c) There are more feature selection strategies can be tested for optimization of external markers layout.

\section{ACKNOWLEDGMENT}

This work is supported by National Key Technology R\&D Program of China under Grant 2013BAI01B01.

\section{REFERENCES}

[1] G. Eason, B. Noble, and I. N. Sneddon, "On certain integrals of LipschitzHankel type involving products of Bessel functions," Phil. Trans. Roy. Soc. London, vol. A247, pp. 529-551, April 1955. (references)

[2] J. Clerk Maxwell, A Treatise on Electricity and Magnetism, 3rd ed., vol. 2. Oxford: Clarendon, 1892, pp.68-73.

[3] I. S. Jacobs and C. P. Bean, "Fine particles, thin films and exchange anisotropy," in Magnetism, vol. III, G. T. Rado and H. Suhl, Eds. New York: Academic, 1963, pp. 271-350.

[4] K. Elissa, "Title of paper if known," unpublished.

[5] R. Nicole, "Title of paper with only first word capitalized," J. Name Stand. Abbrev., in press.

[6] Y. Yorozu, M. Hirano, K. Oka, and Y. Tagawa, "Electron spectroscopy studies on magneto-optical media and plastic substrate interface," IEEE Transl. J. Magn. Japan, vol. 2, pp. 740-741, August 1987 [Digests 9th Annual Conf. Magnetics Japan, p. 301, 1982].

[7] M. Young, The Technical Writer's Handbook. Mill Valley, CA: University Science, 1989.

[8] K. M. Langen, D. T. Jones, Organ motion and its management., International journal of radiation oncology, biology, physics 50 (1) (2001) $265-278$.

[9] P. J. Keall, G. S. Mageras, J. M. Balter, R. S. Emery, M. Forster, S. B. Jiang, J. M. Kapatoes, D. A. Low, J. Murphy, B. R. Murray, et al., The management of respiratory motion in radiation oncology report of aapm task group 76a), Medical physics 33 (10) (2006) 3874-3900.

[10] P. Giraud, A. Houle, Respiratory gating for radiotherapy: main technical aspects and clinical benefits, ISRN Pul-monology 2013.

[11] Y. Seppenwoolde, H. Shirato, K. Kitamura, S. Shimizu, van Herk, J. V. Lebesque, K. Miyasaka, Precise and real-time measurement of $3 \mathrm{~d}$ tumor motion in lung due to breathing and heartbeat, measured during radiotherapy, International Journal of Radiation Oncology* Biology* Physics 53 (4) (2002) 822-834.

[12] H. Shirato, S. Shimizu, K. Kitamura, T. Nishioka, Kagei, S. Hashimoto H. Aoyama, T. Kunieda, N. Shi-nohara, H. Dosaka-Akita, et al., Fourdimensional treat-ment planning and fluoroscopic real-time tumor tracking radiotherapy for moving tumor, International Journal of Radiation Oncology* Biology* Physics 48 (2) (2000) 435-442.
[13] C. Gianoli, M. Riboldi, M. F. Spadea, L. L. Travaini, Ferrari, R. Mei, R. Orecchia, G. Baroni, A multiple points method for 4d ct image sorting, Medical physics 38 (2) (2011) 656-667.

[14] R. Chauvin, M. Hamel, S. Briere, F. Ferland, F. Grondin, Letourneau, M Tousignant, F. Michaud, Contact-Free Respiration Rate Monitoring Using a Pan-Tilt Thermal Camera for Stationary Bike Telerehabilitation Sessions, IEEE Systems Journal 10 (3) (2016) 1046-1055.

[15] J. K. Locklin, J. Yanof, A. Luk, Z. Varro, A. Patriciu, J. Wood, Respiratory biofeedback during ct-guided procedures, Journal of Vascular and Interventional Radi-ology 18 (6) (2007) 749-755.

[16] S. L. Meeks, W. A. Tome,' T. R. Willoughby, P. A. Ku-pelian, T. H. Wagner, J. M. Buatti, F. J. Bova, Optically guided patient positioning techniques, in: Seminars in radiation oncology, Vol. 15, Elsevier, 2005, pp. 192-201.

[17] E. Ford, G. Mageras, E. Yorke, K. Rosenzweig, R. Wag-man, C. Ling, Evaluation of respiratory movement dur-ing gated radiotherapy using film and electronic portal imaging, International Journal of Radiation Oncology* Biology* Physics 52 (2) (2002) 522-531.

[18] G. C. Sharp, S. B. Jiang, S. Shimizu, H. Shirato, Predic-tion of respiratory tumour motion for real-time image-guided radiotherapy, Physics in medicine and biology 49 (3) (2004) 425.

[19] P. S. Verma, H. Wu, M. P. Langer, I. J. Das, G. Sandison, Survey: realtime tumor motion prediction for image-guided radiation treatment, Computing in Science \& Engineering 13 (5) (2011) 24-35.

[20] D. Putra, O. Haas, J. Mills, K. Bumham, Prediction of tumour motion using interacting multiple model filter, in: Advances in Medical, Signal and Information Processing, 2006. MEDSIP 2006. IET 3rd International Conference On, IET, 2006, pp. 1-4.

[21] S. J. Lee, Y. Motai, M. Murphy, Respiratory motion esti-mation with hybrid implementation of extended kalman filter, IEEE Transactions on Industrial Electronics 59 (11) (2012) 4421-4432.

[22] S. J. Lee, Y. Motai, E. Weiss, S. S. Sun, Customized prediction of respiratory motion with clustering from multiple patient interaction, ACM Transactions on In-telligent Systems and Technology (TIST) 4 (4) (2013) 69.

[23] J. Kipritidis, G. Hugo, E. Weiss, J. Williamson, P. J. Keall, Measuring interfraction and intrafraction lung function changes during radiation therapy using four-dimensional cone beam ct ventilation imaging, Medical physics 42 (3) (2015) 1255-1267.

[24] J. Goodband, O. Haas, J. Mills, A comparison of neural network approaches for on-line prediction in igrt, Medi-cal physics 35 (3) (2008) 1113-1122.

[25] M. J. Murphy, D. Pokhrel, Optimization of an adaptive neural network to predict breathing, Medical physics 36 (1) (2009) 40-47.

[26] S. Park, S. J. Lee, E. Weiss, Y. Motai, Intra-and inter-fractional variation prediction of lung tumors using fuzzy deep learning, IEEE journal of translational engineering in health and medicine 4 (2016) 1-12.

[27] Y. Freund, Boosting a weak learning algorithm by ma-jority., in: COLT, Vol. 90, 1990, pp. 202-216.

[28] Y. Freund, R. E. Schapire, et al., Experiments with a new boosting algorithm, in: Icml, Vol. 96, 1996, pp. 148-156.

[29] J. H. Friedman, Greedy function approximation: a gradi-ent boosting machine, Annals of statistics (2001) 1189- 1232.

[30] F. Pedregosa, G. Varoquaux, A. Gramfort, V. Michel, B. Thirion, O. Grisel, M. Blondel, P. Prettenhofer, R. Weiss, V. Dubourg, et al., Scikitlearn: Machine learn-ing in python, Journal of Machine Learning Research 12 (Oct) (2011) 2825-2830.

[31] D. Mease, A. Wyner, Evidence contrary to the statistical view of boosting, Journal of Machine Learning Research 9 (Feb) (2008) 131-156. 